\title{
Tutoria Virtual Baseada em Sistemas Multiagente
}

\author{
Romero Gomes da Silva, UFRR, romero.ufrr@gmail.com \\ Marcelo Batista de Souza, NEaD/UFRR, marcelo.souza@ufrr.br \\ Márcio André Andrade Silva, UFRR, maasilva33@gmail.com
}

Márcio Aurélio dos Santos Alencar, PPGI/UFAM, marcio.alencar@ppgi.ufam.edu.br

\begin{abstract}
Resumo. Com a disseminação da Educação a Distância no Brasil, as oportunidades de acesso ao conhecimento foram ampliadas. Aspectos da vida humana estão sendo copiados para sistemas computacionais inteligentes, $e$ utilizados em processos de ensino-aprendizagem. O artigo apresenta os sistemas Alert@me e MAIN, baseadosem tecnologia multiagente. Ambos são uma alternativa para auxiliar o trabalho da tutoria humana enviando mensagens de texto e e-mail de forma autônoma. A abordagem estimula usuários de um Ambiente Virtual de Aprendizagem, através de notificações, para que interajam e realizem atividades propostas pelo professor. Os sistemas estão na terceira fase de testes e os resultados são promissores.
\end{abstract}

Palavras-chave: Sistemas Multiagente, Ambiente Virtual de Aprendizagem (AVA), Educação a Distância.

\section{Virtual Tutoring Based on Multi-Agent Systems}

\begin{abstract}
With the spread of Distance Education in Brazil, the opportunities of access to knowledge were expanded. Aspects of human life are being copied to intelligent computational systems, and processes used in teaching and learning. The article presents the systems Alert@me and MAIN, based on multi-agent technology. Both are an alternative to assist the work of human tutoring in the sending text messages and email autonomously. The approach encourages users of a Virtual Learning Environment, through notifications, to interact and perform activities proposed by the teacher. The systems are in the third phase of testing and the results are promising.
\end{abstract}

Keywords: Multiagent Systems, Virtual Learning Environment (VLE), eLearning.

\section{Introdução}

Nos últimos anos, as oportunidades de acesso ao conhecimento foram ampliadas em diversas áreas. Segundo dados do Censo da Educação Superior, a modalidade de Educação a Distância (EaD) registrou 992.927 novas matrículas no ano de 2011 (INEP, 2013). Esse crescimento evidenciou problemas enfrentados por estudantes de diferentes cidades do país como a falta de energia, longas distâncias a serem percorridas, Internet de baixa qualidade, espaço físico e laboratórios de informática limitados.

O Ministério da Educação (MEC) destaca que a interatividade entre estudantes, tutores e professores é um dos pilares para garantir a qualidade de um curso, hoje facilitada pelo avanço das Tecnologias de Informação e Comunicação (TIC) (SEED/MEC, 2007). Entretanto, acompanhar as atividades de um curso à distância torna o processo difícil quando as notificações são concentradas apenas no Ambiente Virtual 
de Aprendizagem (AVA). E mais, pesquisas apontam que AVAs possuem limitações evidenciadas nos trabalhos de Alencar e Netto (2012), Silva et al (2013) e Spósito et al (2008), quanto ao suporte a percepção e ao acompanhamento das atividades.

O presente trabalho descreve os Sistemas Multiagente (SMA) Alert@me e MultiAgent INformers (MAIN), integrados a um AVA, utilizado pelo Núcleo de Educação a Distância (NEaD) da Universidade Federal de Roraima (UFRR). O Alert@me monitora o ambiente para notificar usuários sobre a ocorrência de eventos, utilizando Short Message Service (SMS), serviço disponibilizado por operadoras de telefonia móvel. Já o MAIN, utiliza e-mails para estimular usuários a interagir com o ambiente, após observar e avaliar o nível participação de cada um no AVA. A abordagem é uma alternativa para auxiliar o trabalho da tutoria humana no desenvolvimento pedagógico do curso, considerando recursos e serviços tecnológicos dos quais os estudantes possuem nos pólos $\mathrm{EaD}$.

O restante do artigo apresenta os trabalhos correlatos que serviram como referência e estão contribuindo para o desenvolvimento dos sistemas Alert@me e MAIN. $\mathrm{Na}$ seção 3, são destacados os SMAs que atuam no AVA. A seção 4 descreve os resultados alcançados. As conclusões parciais e os trabalhos em andamento são apresentados na seção 5. As referências que sustentam a discussão sobre o tema finalizam o trabalho.

\section{Trabalhos Correlatos}

Nesta seção, serão apresentados os trabalhos correlatos que serviram de base teórica para o desenvolvimento do artigo, dos quais foram extraídos conceitos e abordagens utilizadas na construção dos sistemas Alert@me e MAIN.

Almeida et al (2012) apontaram que os AVAs não oferecem ferramentas ágeis de monitoramento. Os autores utilizaram o ambiente Tidia-AE, e apresentaram algumas configurações de alertas automatizados em AVAs que podem apoiar a tutoria. Lim et al (2011) utilizaram SMS para auxiliar no gerenciamento e motivação de estudantes, ao desenvolverem suas atividades. Os autores relataram que a abordagem contribuiu para diminuir as dificuldades enfrentadas na modalidade EaD, além de estreitar a comunicação do estudante com a universidade.

No trabalho de Wilges et al (2010) um SMA mapeou a evasão na EaD. Os autores apresentaram um modelo para combater o problema que melhorou a eficácia do sistema e enfatizaram o uso dessa tecnologia em AVAs. Lyra e Santos (2012) apresentaram um SMA capaz de monitorar a frequência dos estudantes e as datas de envio dos trabalhos na plataforma Moodle, bem como a estrutura do seu Banco de Dados (BD), utilizando envio de mensagens para evitar o problema da evasão e perda de prazos com relação ao cumprimento das atividades do curso.

\section{Monitoramento do AVA para Apoiar o Trabalho da Tutoria Humana}

Os sistemas foram desenvolvidos baseado em SMA com a finalidade de apoiar o papel do tutor. Para tanto, observações feitas pela coordenação de tutoria e relatos de tutores do NEaD/UFRR, colaboraram para que tarefas executadas no AVA fossem projetadas noAlert@me e MAIN. 


\subsection{Sistemas Multiagente: Alert@me e MAIN}

Os SMAs foram concebidos para oferecer suporte ao trabalho da tutoria humana com a proposta de melhorar a qualidade da comunicação, cumprimento das obrigações e desempenho do estudante ao longo de um curso EaD. O Alert@me monitora o ambiente Moodle para notificar seus usuários sobre a ocorrência de eventos através de SMS (popularmente chamado de "torpedo"), serviço disponibilizado por operadoras de telefonia móvel.

O MAIN complementa a atuação do Alert@me. Ele realiza o monitoramento das interações para observar e avaliar em que nível elas estão ocorrendo, além de estimular os usuários do AVA através de e-mails, com a finalidade de que tenham efetiva participação no processo de ensino-aprendizagem. Ambos tiveram o seu desenvolvimento baseado na Methodology MultiAgent Systems Engineering (MaSE), proposta por DeLoach (2001). Conforme Silva et al (2007) destacam, a metodologia MaSE possibilita coerência entre modelos, criação de SMAs e desenvolvimento de sistemas que satisfaçam os objetivos iniciais do projeto. A Figura 1 apresenta um diagrama que identifica a especificação inicial do Alert@me e MAIN, contendo o objetivo geral e sub-objetivos do sistema.

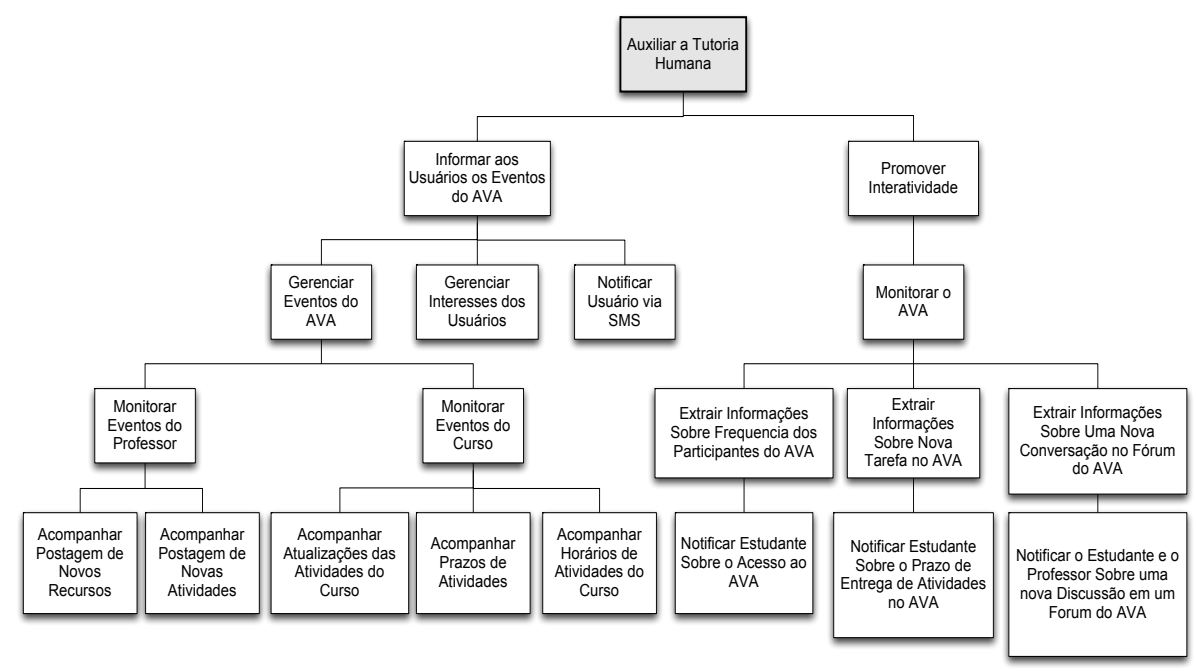

Figura 1 - Diagrama de Hierarquia de Objetivos unificado dos sistemas.

Na Figura 1, o conjunto de sub-objetivos visa estimular a interatividade entre os usuários do AVA Moodle, explorando o envio de SMS e e-mail para informá-los sobre a ocorrência de eventos como recursos (página de texto simples, página web, arquivo ou site e diretório), atividades (banco de dados, chat, escolha, fórum, glossário, lição, pesquisa de avaliação, questionário e wiki), tarefas (modalidade avançada de carregamento de arquivos, texto online, envio de arquivo único e atividade offline), prazos de encerramento, horários de atividades e nível de ausência dos estudantes no ambiente.

Segundo DeLoach (2001), o cumprimento desse conjunto de sub-objetivos permite alcançar o objetivo principal do sistema. Para atingi-los foi definida uma arquitetura composta por recursos de software e hardware. A arquitetura geral dos sistemas está sendo apresentada na Figura 2, com destaque para os seus principais componentes e a atuação da sociedade de agentes que constituem o SMA. 


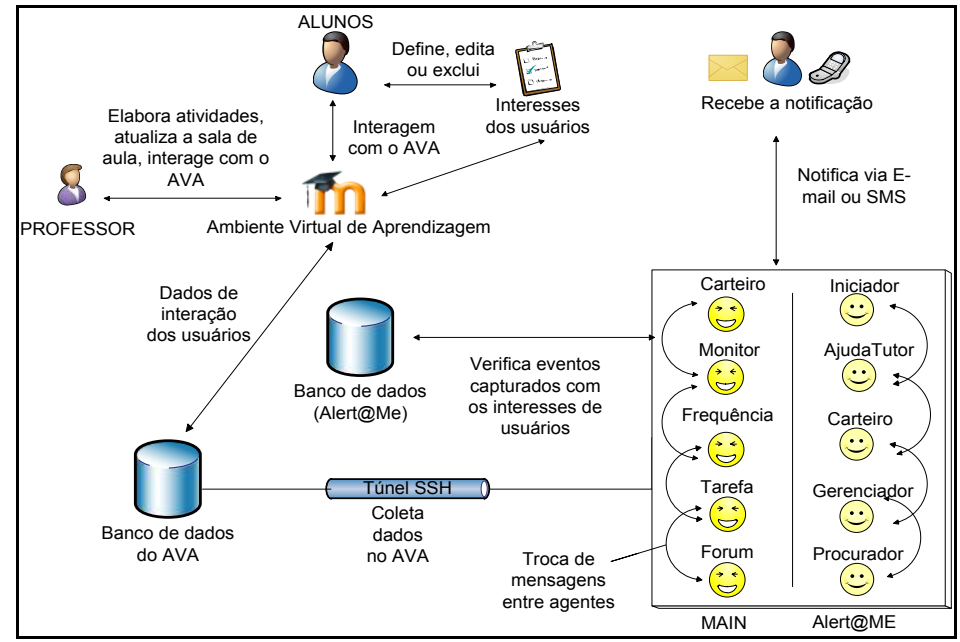

Figura 2 - Arquitetura conjunta dos sistemas Alert@me e MAIN.

Os recursos de hardware e software mostrados na Figura 2 proporcionam a comunicação entre usuários do AVA e os SMAs Alert@me e MAIN. Os comportamentos de cada um deles serão destacados nas seções seguintes.

\subsubsection{Comportamento dos Agentes Inteligentes do Alert@me}

- Iniciador - Estabelece e gerencia a conexão segura com o BD do AVA Moodle utilizando protocolo Secure Shell (SSH), devido as políticas de segurança adotadas pela UFRR Além de informar o status da conexão para os agentes Ajuda Tutor, Carteiro, Gerenciador e Procurador;

- Ajuda Tutor - Identifica e informa aos usuários sobre horários de atividades, prorrogação de prazos e encerramento de tarefas com data e hora;

- Carteiro - Estabelece e gerencia conexão com um Modem $3 G$ (utilizado para transmitir a notificação). Envia SMS contendo as informações recebidas pelo agente Gerenciador, sobre o evento ocorrido no ambiente aos aparelhos celulares dos usuários. Ou, retransmite em caso de falha na transmissão;

- Gerenciador - Gerencia e analisa os interesses pessoais de cada usuário com os eventos encontrados pelo agente Procurador. E, formata uma mensagem de texto baseado em 03 (três) elementos de percepção ("O que", "Quem", "Onde"), repassando a mensagem para o agente Carteiro.

- Procurador - Identifica a postagem de novos recursos, atividades e tarefas que ocorrem no AVA Moodle, repassando estas informações ao agente Gerenciador.

Também foi desenvolvido um módulo Moodle, chamado alert@me. Ele disponibiliza um formulário web contendo notificação sobre recursos, tarefas e prazos de encerramento de atividades. O módulo permite selecionar, salvar, editar e excluir tópicos de interesse. Para acessá-lo, o professor/tutor disponibiliza o módulo na sala de aula e os usuários cadastram um número de telefone celular e seus interesses.

O Alert@me monitora o AVA Moodle em busca de ocorrência de eventos, realizando uma analise entre a lista de interesses pessoais do usuário (definida através do móduloalert@me) com os eventos encontrados pelo SMA. Por fim, o sistema envia notificações aos usuários via SMS. Alguns exemplos de mensagens do Alert@me estão sendo apresentados na Figura 3. 

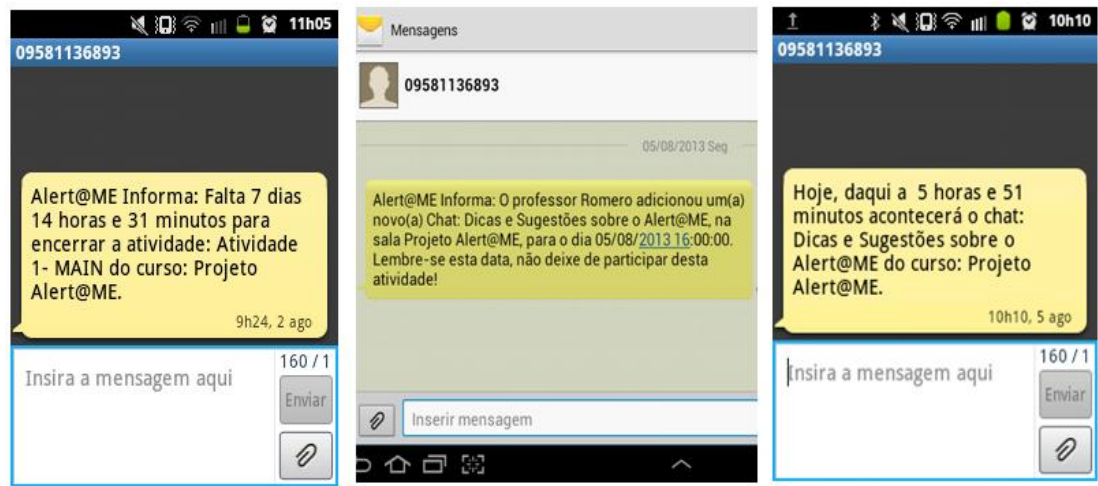

Figura 3 - Exemplos de mensagens de texto enviada pelo SMAAlert@me.

A primeira mensagem informa sobre o prazo de encerramento de uma atividade em um curso. A segunda apresenta uma notificação relatando que o professor adicionou uma atividade chat em um curso com data e horário, seguido de uma frase motivacional. Por fim, um exemplo de notificação que alerta o usuário sobre o horário que ocorrerá uma atividade.

\subsubsection{Comportamento dos Agentes Inteligentes do $M A I N$}

- Carteiro - Seleciona e envia correio eletrônico (e-mail) para os usuários. O conteúdo do e-mail é estabelecido pela análise das informações recebidas do agente Monitor e um banco de mensagens pré-determinadas para cada tipo ocorrência (fórum, atividades e frequência).

- Monitor - Gerencia o desempenho dos estudantes, através de informações recebidas dos agentes Frequência, Tarefa e Fórum. Ele analisa dados anteriores e atuais dos estudantes, repassando informações relevantes ao agente Carteiro.

- Frequência - Monitora e determina nível de ausência do aluno no AVA, classificando em três categorias - "Grande Abandono" (15 a 30 dias), "Médio Abandono" (07 a 15 dias) e "Pequeno Abandono" (01 a 07 dias). Ele comunica estas informações ao agente Monitor.

- Tarefa - Verifica a postagem de novas tarefas de envio de arquivo único na sala virtual. Ele comunica ao agente Monitor informações sobre a atividade (prazo e data limite para entrega).

- $\quad$ Fórum - Acompanha a publicação de fóruns de discussão e postagem de novas mensagens. Comunica ao agente Monitor informações sobre mensagens (autoria, data e hora).

O propósito inicial do MAIN era monitorar a participação dos usuários em fóruns de discussão para avaliar a qualidade das interações e promover a interatividade no AVA. Durante a pesquisa, houve a necessidade de ampliar a atuação do sistema enviar e-mails baseado na lista de interesses do usuário, apresentada na Figura 3. A estratégia foi adotada pois não há garantias de que todos os usuários possuam telefone celular e, caso tenham, se estarão funcionando no momento do envio da notificação via SMS. Nas Figuras 4 e 5, estão sendo apresentados exemplos de mensagens enviadas pelo SMA MAIN a usuários do AVA. 


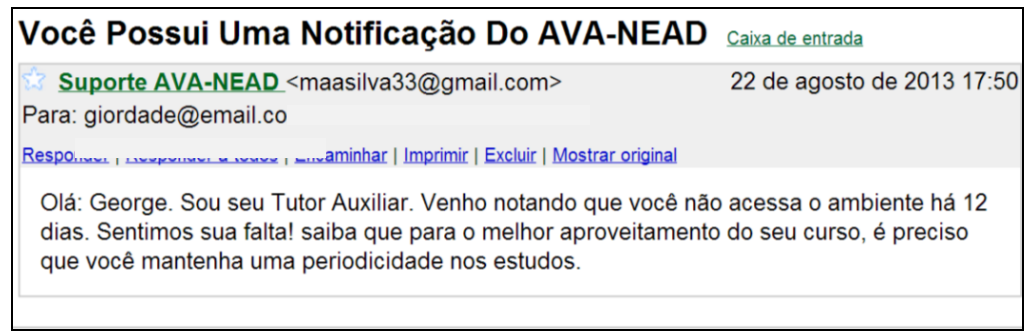

Figura 4 - Exemplo 1 de mensagem de e-mail enviada pelo SMA MAIN.

A Figura 4 apresenta um exemplo de e-mail referente ao nível de frequência do aluno no ambiente. $\mathrm{O}$ conteúdo da mensagem informa que há 12 dias um aluno não acessa o AVA, caracterizando um "Médio Abandono" (07 a 15 dias). Além de uma frase motivacional para estimular a interatividade com o AVA.

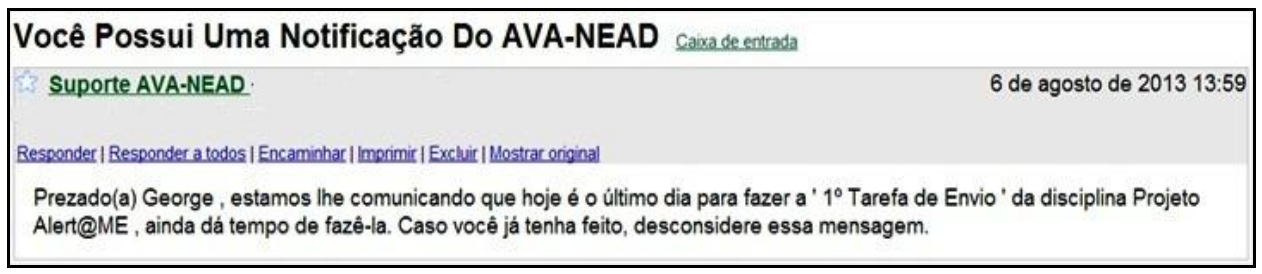

Figura 5 - Exemplo 2 de mensagem de e-mail enviada pelo SMA MAIN.

A Figura 5 apresenta outro exemplo de e-mail enviado pelo MAIN. Neste caso, o conteúdo da mensagem informa o nome e o prazo para entregar a atividade publicada em um determinado curso, juntamente com uma frase motivacional para que o aluno não deixe de fazer a atividade proposta.

\section{Testes e Resultados}

Nesta seção serão apresentados os testes e os resultados obtidos na integração dos sistemas. Os principais softwares utilizados no seu desenvolvimento e execução foram o AVA Moodle 1.9.17+, o JADE 4.2, linguagem de programação Java, ferramenta agentTool, biblioteca SMSLib 3.5.2, JSCh 1.0, JavaMail e Hibernate 4.2. A abordagem na implementação foi a prototipação. A figura 6 apresenta a troca de mensagem entre os agentes. No entanto, foram realizados os testes para detectar a presença de falhas no sistema. Os registros contidos no $\mathrm{BD}$ e os relatos dos envolvidos na pesquisa colaboraram para que fossem corrigidas.

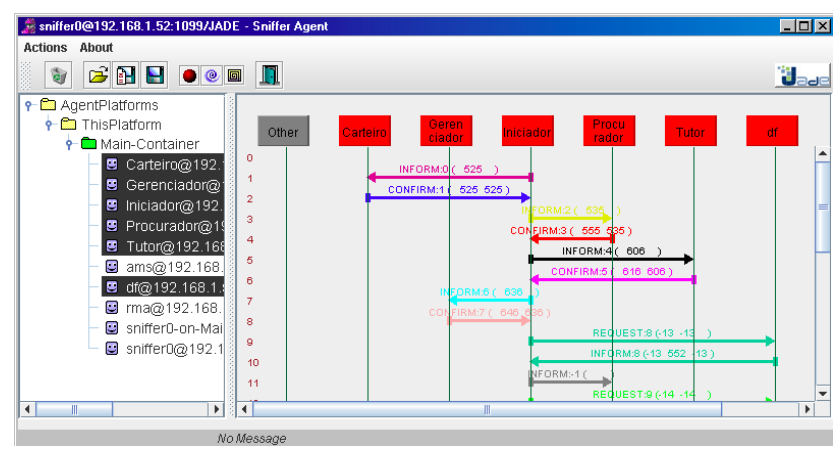

Figura 6 - Troca de mensagens entre os agentes.

Nos testes realizados com o Alert@me, foi utilizado uma sala virtual do ambiente Moodle do NEaD/UFRR composta por 2 (dois) professores e 8 (oito) 
estudantes escolhidos aleatoriamente, onde $63 \%$ deles participaram efetivamente dos testes sem apresentar problemas. Do grupo dos 37\% restantes, $12 \%$ atualizaram o celular fora do padrão adotado pelo SMA, 13\% já possuíam cadastro atualizado, porém com formato de dados incorretos, e $12 \%$ deles não atualizaram o número de telefone celular. Quanto ao envio das notificações, foi utilizando um Modem $3 G$ Huawei E226 em conjunto com a biblioteca SMSLib para realizar a transmissão das SMS. O Alert@me gerou 146 (cento e quarenta e seis) mensagens de texto referentes a publicação de recursos, atividades, prazos de encerramento e horário de entrega de tarefas. Na Figura 7 estão sendo mostrados os resultados.

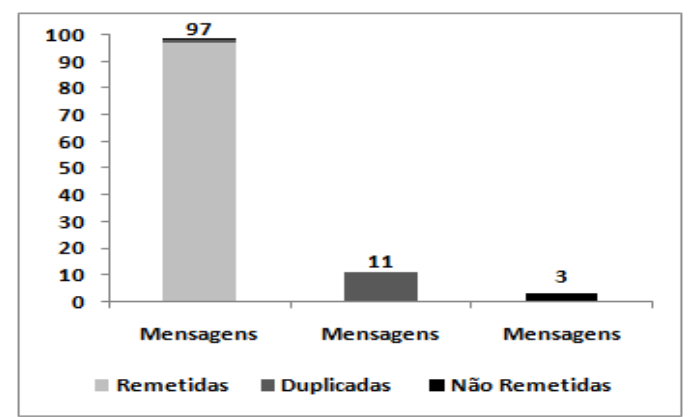

Figura 7 - Notificações enviadas pelo SMA Alert@me.

É possível observar que $97 \%$ das mensagens foram enviadas com sucesso. $\mathrm{O}$ restante, $3 \%$ delas não puderam ser entregues devido ao número de telefone celular dos usuários estarem em um formato diferente do padrão adotado pelo sistema. Vale ressaltar que, devido às falhas encontradas durante os testes, $11 \%$ das mensagens de texto enviadas, foram repetidas.

Os testes em relação ao cadastramento da lista de interesses mostram que $62,5 \%$ obtiveram sucesso. Os demais usuários não registraram as suas escolhas no AVA. Foram analisados casos como a postagem de recursos e atividades, onde o sistema obteve êxito ao perceber a ocorrência desses eventos. O SMA realizou corretamente a análise da lista pessoal de interesse capturada no ambiente Moodle. Entretanto, nos casos de acompanhamento de horários e prorrogação dos prazos de atividades, houve duplicidade no envio da notificação aos usuários. O problema está sendo analisado para ser corrigido nas próximas versões.

Os testes realizados com o sistema MAIN foram feitos com os usuários da mesma sala virtual do ambiente Moodle. Responderam ao questionário $100 \%$ dos participantes da pesquisa. O levantamento dos dados foi realizado com o intuito de descobrir qual o dispositivo utilizado pelos usuários para acessar o AVA, com que frequência eles fizeram isso, descobrir se os usuário tiveram dificuldades para realizar as atividades e se acessaram a caixa de e-mail regularmente para verificar o recebimento de novas mensagens. Os resultados estão evidenciados na Figura $8 \mathrm{a}$ e $8 \mathrm{~b}$.

Os resultados da Figura 8a apontam que a maioria dos usuários ainda utiliza o Personal Computer (PC) para acessar o AVA, mas destaca a tendência da utilização do aparelho celular. Com relação à frequência de acesso dos usuários, os números apresentaram um resultado parelho. O destaque negativo ficou por conta dos $30 \%$ que pouco acessaram o AVA durante a fase de testes. Os resultados da avaliação referente à Figura $8 \mathrm{~b}$ apontaram que a maioria dos usuários conseguiram fazer suas atividades em tempo hábil. O ponto positivo é o registro de que todos possuíam endereços de e-mail. 
Desse grupo o destaque foi para os $80 \%$ de usuários que verificam as mensagens diariamente.
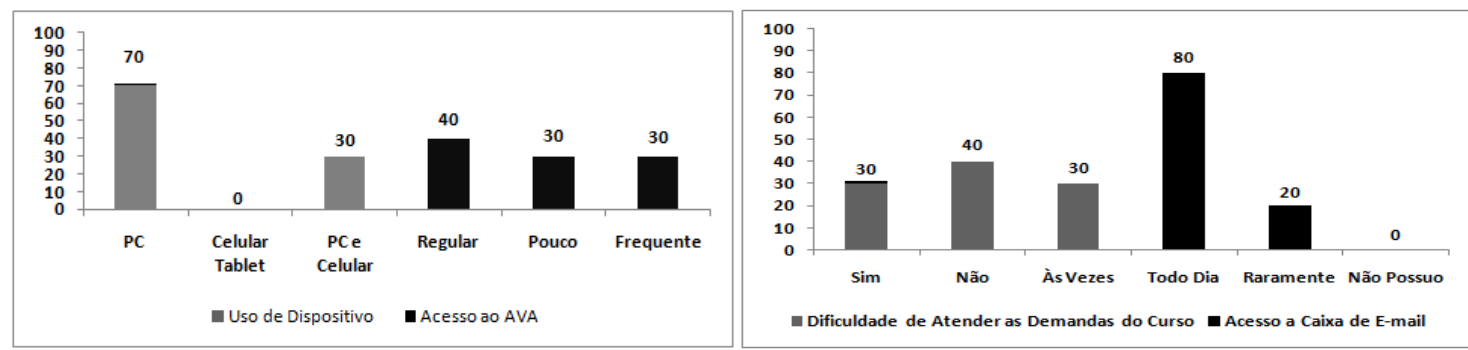

Figura 8 - a) Tipo de dispositivo e frequência no acesso ao AVA e b) Realização de atividades e verificação de mensagens na caixa de e-mail.

Os testes colaboraram para que os protótipos fossem melhorados, através de ajustes no código dos agentes. Isto aumentou a qualidade e usabilidade dos sistemas. Também foram empregados Testes Não-Estruturados (TNE) sem a adoção de grupos específicos de usuários. Os participantes da pesquisa tinham características heterogêneas relativas à idade e ao conhecimento sobre o assunto. As etapas dos testes compreenderam no desenvolvimento e na disponibilização dos protótipos acessado pela rede local e Internet.

Os resultados dos TNEs apontaram inúmeras falhas nos protótipos por conta de incompatibilidade de sistema e linguagens utilizadas. A medida que iam surgindo as falhas, elas eram avaliadas e corrigidas. Os resultados dos testes e TNEs foram satisfatórios, pois apontaram a necessidade de ajustes nos protótipos para proporcionar auxílio ao trabalho da tutoria, mediado por tecnologia SMA.

\section{Conclusões e Trabalhos em Andamento}

Neste trabalho foi apresentada uma abordagem para o uso de SMAs em um AVA. Os sistemas Alert@me e MAIN foram desenvolvidos com a finalidade de apoiar o papel do tutor no AVA Moodle. A estratégia adotada foi monitorar ambiente virtual e utilizar o serviço de envio de mensagens (e-mail e SMS), baseado em uma lista de interesses, para informar a ocorrência de eventos no ambiente, auxiliando o trabalho da tutoria humana e aproximando estudantes de um curso $\mathrm{EaD}$ em seu processo de ensino-aprendizagem.

Após o levantamento sobre o estado da arte, foi definida a estrutura utilizada nos sistemas baseado em diferentes abordagens e emprego de tecnologias. Com a pesquisa, já foi possível desenvolver parcialmente os sistemas pautado em duas coisas. A primeira delas está relacionada com o reuso de códigos e integração de tecnologias na implementação. A segunda se refere à adoção e uso de softwares open source que permitiram a alteração e até inclusão de códigos.

No desenvolvimento dos protótipos, se utilizou o AVA Moodle, portanto suas ferramentas não foram incluídas nos testes, visto que já existe na literatura exposição sobre elas. Os testes exploraram basicamente o envio de mensagens para auxiliar a tutoria humana com a proposta de melhorar a qualidade da comunicação, cumprimento das obrigações e desempenho do estudante ao longo de um curso EaD. Os resultados apresentados serviram de insumos para aperfeiçoamento dos protótipos nos quesitos de usabilidade e qualidade do sistema.

A arquitetura e o comportamento dos agentes foram definidos com base em observações feitas pela coordenação de tutoria e relatos de tutores do NEaD/UFRR. A 
partir daí, os casos de uso foram construídos. A Unified Modeling Language (UML) foi utilizada para identificar em alto nível as funcionalidades dos sistemas. A metodologia MaSE permitiu analisar e modelar os SMAs, que por meio de seus modelos possibilitou descrever os tipos de agentes, as interfaces da interação entre eles e a arquitetura de cada um. Isto garantiu estruturar, documentar e atingir o objetivo principal dos sistemas.

Em geral, os AVAs utilizados na $\mathrm{EaD}$ dispõe de um serviço de monitoramento limitado. Além disso, eles restringem a informação sobre a ocorrência de eventos para acesso somente dentro da sala virtual. Isto dificulta a participação de usuários e os obriga a estarem conectados ao ambiente. Em relação ao custo/benefício do SMS, a motivação para adotar o serviço se originou em decorrência da sua fácil utilização, amplo alcance geográfico devido às redes móveis, popularidade entre seus usuários, além de ser suportada pela maioria dos aparelhos que utilizam a tecnologia Global System for Mobile Communications (GSM).

No entanto, o emprego dessa tecnologia no Alert@me agrega custos financeiros e sua viabilidade dependerá de planos oferecidos por operadoras do setor. Quanto ao MAIN, o sistema surgiu com a necessidade de alcançar também os usuários do AVA que não possuem aparelho de telefone celular. O desenvolvimento de 2 (dois) SMAs distintos ajudou a manter o foco dos objetivos específicos de cada um.

Para trabalhos futuros pretende-se desenvolver uma ontologia própria para os sistemasAlert@me e MAIN, continuar a fase de testes exaustivos, ajustar o código com o objetivo de especializar as ações dos agentes e realizar um estudo que aborde a questão do delay, capaz de apontar o tempo gasto no envio/recebimento de mensagens.

Para finalizar, a abordagem proposta no artigo se apresenta como flexível, extensível, escalável, baseado em agentes, autônoma, aberta e livre para sistemas que têm a finalidade de auxiliar usuários em AVAs. Os comportamentos projetados para os agentes, os tornaram capazes de perceberem e atuarem no ambiente, ora agindo, ora reagindo, ora interagindo, ora sugerindo alguma ação para o usuário.

A idéia de disponibilizar o AVA Moodle sendo mediado por tecnologia SMA permitiu a sua automatização através de consultas ao BD e envio de mensagens pelos agentes como um recurso para auxiliar o usuário no desenvolvimento das suas atividades, conforme evidenciado nos trabalhos de Passerino et al (2007) e Souza (2013).

\section{Referências Biblográficas}

ALENCAR, M. A. S.; NETTO, J. F. M. (2012). Sistema Multiagente para Apoiar a Percepção e o Acompanhamento de Atividades em Ambientes Virtuais de Aprendizagem. In: XXIII SIMPÓSIO BRASILEIRO DE INFORMÁTICA NA EDUCAÇÃO, 2012, Rio de Janeiro, RJ. Anais do XXIII Simpósio Brasileiro de Informática na Educação, 2012.

ALMEIDA, A.; PIMENTEL, E. P.; STIUBIENER, I. (2012). Estratégias para o Monitoramento de Ações de Tutoria na Educação a Distância. In: WORKSHOP SOBRE AVALIAÇÃO DE ACOMPANHAMENTO DA APRENDIZAGEM EM AMBIENTES VIRTUAIS, 5., Rio de Janeiro. In: Anais do I Congresso Brasileiro de Informática na Educação.

DELOACH, S. A. (2001). Analysis and Design using MaSE and agentTool. In: PROCEEDINGS OF THE 12TH MIDWEST ARTIFICIAL INTELLIGENCE 
AND COGNITIVE SCIENCE CONFERENCE - MAICS, 2001. Miami University, Oxford, Ohio, March 31 - April 1, 2001.

Instituto Nacional de Estudos e Pesquisas Educacionais Anísio Teixeira - INEP (2011). Censo da Educação Superior. Disponível em http://portal.inep.gov.br/web/censo-daeducacao-superior. Acessado em 15/03/2013.

LIM, T.; FADZIL, M.; MANSOR, N. (2011). Mobile Learning via SMS at Open University Malaysia: Equitable, Effective, and Sustainable. In: The Internacional Review of Research in Open and Distance Learning. Vol. 12, N. 2, ISSN. 14923831, February.

LYRA, F.; SANTOS, N. (2012). Agentes de Software no Monitoramento de Alunos em Educação a Distância. In: XVIII WORKSHOP DE INFORMÁTICA NA ESCOLA, Rio de Janeiro/RJ.

Secretaria de Educação a Distância do Ministério da Educação - SEED/MEC (2007). Referências de Qualidade para a Educação Superior a Distância. Disponível em http://portal.mec.gov.br/seed/arquivos/pdf/legislacao/refead1.pdf. Acessado em 15/03/2013.

SILVA, J. M. C.; BAVARESCO, N.; SILVEIRA, R. A. (2007). Proposta de um Sistema Multi- agentes para a aplicação de Objetos Inteligentes de Aprendizagem Seguindo o Padrão SCORM. In: XVIII SIMPÓSIO BRASILEIRO DE INFORMÁTICA NA EDUCAÇÃO, São Paulo/SP. Porto Alegre: SBC - Sociedade Brasileira de Computação. v. 1. p. 175-185, 2007.

SILVA, R. G.; SOUZA, M. B.; ALMEIDA, T. O. (2013). Alert@me, um Sistema de Notificação Inteligente que Utiliza Ambiente Virtual de Aprendizagem, Tecnologia Multiagente e Serviço de Mensagens Curtas. In: III ESCOLA REGIONAL DE INFORMÁTICA DA REGIÃO NORTE I - ERIN, 2013, Boa Vista - RR. Porto Alegre: SBC, 2013.

SOUZA, M. B. (2013). Percepções e Ações da Coordenação de Tutoria no Desenvolvimento Pedagógico do Curso. In: ESUD 2013 - X CONGRESSO BRASILEIRO DE ENSINO SUPERIOR A DISTÂNCIA - Belém - PA. 11 - 13 de junho de 2013 - UNIREDE.

SPÓSITO, M. A. F.; CASTRO, T. H. C.; JUNIOR, A. N. C (2008). Estação de Percepção: Uma Abordagem para o Monitoramento em Ambientes Virtuais de Aprendizagem. In: SIMPÓSIO BRASILEIRO DE INFORMÁTICA NA EDUCAÇÃO. 19, Fortaleza, 2008.

PASSERINO, L. M.; GLUZ, J. C.; VICARI, R. M. (2007). Uma Proposta para Mediação Tecnológica em Espaços Virtuais de Aprendizagem. In: XVIII SIMPÓSIO BRASILEIRO DE INFORMÁTICA NA EDUCAÇÃO. v. 1. p. 36-7, São Paulo.

WILGES, B.; RIBAS, J. C. S.; CATAPAN, A. H.; BASTOS R. C. (2010). Sistemas Multiagentes: Mapeando a Evasão na Educação a Distância. Revista Novas Tecnologias na Educação - RENOTE, Porto Alegre, v. 8, p.1-10. 